\section{USE OF A MECHANICAL MODEL TO CONVEY THE IDEA OF ENERGETIC COUPLING}

I deal here with a severe deficiency that readily arises in the understanding of the coupling between two chemical reactions, or between a chemical reaction and a transport across a membrane. This difficulty occurs mainly among biologists who have taken only a course or two in biochemistry with a minimum of physical chemical preparation, although one has to suspect an occasional defect in the clarity with which the matter is perceived by more experienced biochemists.

Let me illustrate the difficulty in its most absurd form in this way: The student may get the idea that an energy-requiring reaction can receive its energy if only ATP is being hydrolyzed in the same solution. Or more likely he gets the idea that a discrete package of energy and nothing else is delivered to the reaction on the hydrolysis of ATP. Mitchell calls attention to a statement by a prominent enzymologist that the endergonic reaction may receive only energy and no material from the exergonic one, even though we are restricted to isothermal conditions at the ground state. Gregoria Weber has chosen to bring the matter to attention by challenging audiences with his sentence:

"Every chemical compound generated in metabolism is the result of a reaction which runs towards thermodynamic equilibrium with complete independence of any other chemical reaction occurring at the same time."

Several of our textbooks seem to throw a barrier in the way of the student by defining coupling in one way "two reactions in which the product of one becomes a reactant in the other", and then using the term in quite another, the conventional way. This conventional use of the term I can illustrate by a ligase reaction in which two substances $M$ and $N$ are joined to form $M N$ by coupling with the cleavage of ATP to form AMP + PPi. The coupled reaction takes the course.

$$
\begin{aligned}
& \mathbf{M}+\mathbf{A T P} \rightarrow \mathbf{A M P} \cdot \mathbf{M}+\mathbf{P P}_{\mathbf{i}} \\
& \mathbf{A M P} \cdot \mathbf{M}+\mathbf{N} \rightarrow \mathbf{M N}+\mathbf{A M P}
\end{aligned}
$$

We say then that the cleavage of ATP has been energetically coupled to the synthesis of MN. As an incidental matter we see here two new reactions which are sequentially linked. I believe the key point, however, is that the breakdown of ATP and the synthesis of $M N$ have been fused into a single reaction course, so that the originally perceived reactions, ATP $\rightarrow \mathbf{A M P} \rightarrow \mathbf{P P i}$ and $\mathbf{M N} \rightarrow \mathbf{M}+$ $N$, are largely avoided, unless, of course there is a degree of uncoupling.

Active transport has become so familiar a manifestation of energetic coupling that we can actually use transport to help the student understand the distinction between the concept of coupling and that of sequential linkage. A chemical reaction may supply a substance which subsequently moves across a membrane. Or a chemical reaction may tend to eliminate the substance after it has entered a compartment by crossing a membrane. In either case the chemical reaction can sustain the transport by "pushing" it (supplying the migrating species) or by "pulling" it (removing the species after it has migrated). Although the reaction and the transport step are in sequential linkage, we would never call the process as described an active transport. We decline to call it that because we say there really is no direct energetic coupling of the reaction to the transport process. Just as for the synthesis of $\mathrm{MN}$ coupled with the breakdown of ATP, we require that the chemical reaction and the transport have become fused into one process, which we can represent as follows:

$A \rightarrow B$ (exergonic) fused with $X_{1} \rightarrow X_{2}$ (transfer of $X$ from phase 1 to phase 2 against a gradient), yielding:

$$
A+X_{1} \rightarrow B+X_{2}
$$

\section{HALVOR N. CHRISTENSEN}

Department of Biological Chemistry,

The University of Michigan

Ann Arbor

Michigan 48109, U.S.A.

(Unfortunately our symbolism makes no proposal here as to the mechanism of the fusion; but we are to understand that little or no $A \rightarrow B$ or $X_{2} \rightarrow X_{1}$ is now to occur as a separate event, unless an uncoupler is added.)

If we maintain this same distinction between the energetic coupling of one chemical reaction to another, and a mere sequential relation between them, we will be defining coupling in the way most of us use the term in subsequent instruction.

What $I$ like to do in making this point is to show the student a model of the mechanical paradox illustrated in the figure. The rotor is composed of two $10 \mathrm{~cm}$ plastic funnels whose mouths have been sealed together with epoxy cement. The stems are trimmed to a length of perhaps a centimetre. An inclined plane is formed from two $1 \mathrm{~cm}$ softwood panels about $35 \mathrm{~cm}$ long and $9 \mathrm{~cm}$ high at one end, $15 \mathrm{~cm}$ at the other, nailed into a converging relation, as pictured. Two pieces of wood separate the two side panels, one by $3 \mathrm{~cm}$ at the lower end, the other by $23 \mathrm{~cm}$ at the upper end. These two pieces of wood are placed or notched so as to allow space for the rotor to approach the ends of the inclined plane. The difference in the height of each side panel at its two ends is $6 \mathrm{~cm}$. I also use in the demonstration a paper cylinder about $25 \mathrm{~cm}$ long and up to $10 \mathrm{~cm}$ in diameter, and a softwood board of similar length, roughly $2 \times 6 \mathrm{~cm}$ in thickness and width. My commentary runs somewhat as follows:

"Let the difference in the levels at the two ends of this inclined plane represent the difference in the concentration of a solute on the two sides of a membrane. Note that a paper cylinder rolls down this slope with high spontaneity. It will not roll up the slope by itself. Hence we are left with two disadvantages: excessive spontaneity in one direction; and no choice as to direction.

"I'll use this board [on edge] to prop the device at one end so that the plane becomes horizontal. Now note that this rotor with its conical axes roles in one direction with high spontaneity. I want this motion to represent the progress of an exergonic chemical reaction: high spontaneity in one direction, and again no choice in direction.

"Now if we remove the shim we can imagine that we have the reaction occurring so that transport is also produced; i.e. the reaction is taking place in a heterogeneous system. The rotor surprises us by rolling up, not down, the inclined plane. By that motion we will represent active transport.

"When we look closely we understand why the motion is in that direction. In addition to the slope by which we have agreed to represent the concentration gradient, we also see another gradient represented by the shape of the rotor and that of the inclined plane, which together produce the spontaneity we attribute to the chemical reaction. This gradient provides the driving force for the transport;

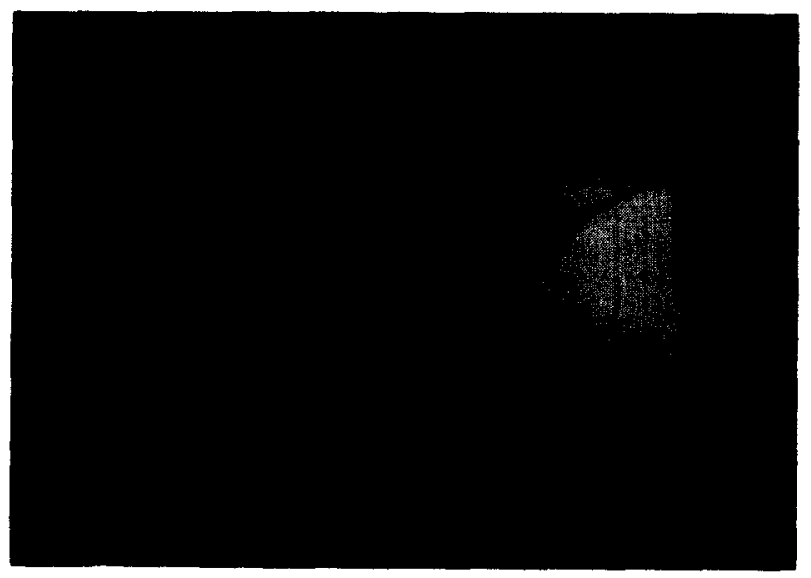


it represents the free energy change of the exergonic reaction. Remember, the slope of the inclined plane represents the free energy change of the endergonic direction of the transport. We will represent, then, the total free energy change of the coupled process by the rise or fall of the centre of gravity of our rotor. We are not surprised to discover that the spontaneous direction of its motion is the one that allows the centre of gravity to move downward."

At this point I look inquiringly at the students. If necessary I ask one of them, "Is something troubling you?"' Usually I find one who will complain. "Dr. C, I think your model may be fraudulent. You say that it will show the coupling between a chemical reaction and a transport, and you have made us feel that there are two motions here to be coupled, one to represent the chemical reaction and one to represent the transport. But what you have there in your hand is a single rotor, and it makes a single motion, namely in the downward direction. You have made us feel that process represents two events, one with a certain degree of spontaneity and the other which must be driven; but this differentiation is only a trick of the mind."

My response: "John, you've grasped precisely the concept of energetic coupling. We begin by thinking of two processes, but when coupling has occurred we have just one motion of flow; the two separate processes have disappeared into one, and survive only to the extent that the coupling is incomplete. We can calculate $\Delta G$ of the two hypothetical components; but these two hypothetical components in no way survive as part of the coupled mechanism."

I then continue, "The model can equally well represent the coupling of two chemical reactions. This model does share a trait with active transport: Even though the two components of the net downward movement of the rotor have been fused into one motion, our attention is still captured by the paradoxical uphill aspect of the migration, just as we see something intuitively wrong about the movement of a solute against its concentration gradient.

"Note also that a small tilt will reverse the direction of spontaneity. [Insert the board under the model so as to intensify the gradient.] In the usual position of the model, the chemical reaction is seen driving the transport. After the shim is inserted, the transport may equally well drive the chemical reaction. Here we have an important feature of active transport across biological membranes. Forward or backward, the two processes are the same fused complex. It is now a familiar relation that a transport which appears to be strongly "downhill" may actually be doing work by driving a chemical reaction, and therefore much less spontaneous than we suppose."

The reader will I think be able to see how a brief presentation of this sort can be encapsulated into an opening lecture on bioenergetics. Other aspects of the discussion will of course need to relate to and reinforce the presentation on coupling if it is to be effective.

\section{Handbook of Organic Reagents in Inorganic Analysis}

Z. Holzbecher, L. Divis, M. Kral, L. Sucha and F. Vlacil, Ellis Horwood Series on Analytical Chemistry, J. Wiley and Sons, 1976. Pp. 734, $£ 19.00$

This is one of 15 volumes edited by Dr. R. A. Chalmers and devoted to analytical chemistry. At a time when courses on this subject are practically non-existent in many universities the series as a whole is very much to be welcomed. Each year numerous papers are published on the applications of organic reagents for inorganic analysis and continuous appraisal and reappraisal of the area is called for. At present there are few monographs listing adequately the various applications, which is the purpose of the present volume.

Five authors have contributed. The first 182 pages are devoted to such topics as the nature of chemical bonds, stereochemistry, isomerism, absorption spectra, equilibria etc. All these topics are more than adequately covered elsewhere, and seem somewhat unnecessary. Chapter 4 (pp. 183-390) dealing with applications of organic reagents in inorganic analysis is somewhat nearer the subject under dircussion. Chapter 5 commencing p. 391 (to the end) is I foel the real thing, and what most people will be looking for and value most in this volume. In this chapter there is a necessarily selective cataloguing of reagents grouped together under different non-metallic as well as metallic elements. The compilation given appears under column headinga, reagents, method, conditions, range (of concentrations detectable), remarks, and references. Spectrophotometric methods are particularly prominent. Posalble interterences are listed.

Even in these days of expensive instrumentation the use of organic reagents has a wide usige, and as a ready source of available procotures this book will be of immense value.
International Newaletter of Chemical Bducation No. 5 Decomber 1976

Produced by the IUPAC Committee on Teaching of Chemistry, with financial support from UNESCO. Available free-of-charge from the IUPAC Secretariat, Bank Court Chambers, 2-3 Pound Way, Cowley Centre, Oxford, OX4 3YF, U.K.

Most member countries of the International Union of Pure and Applied Chemistry (IUPAC) have appointed National Representatives on the Teaching of Chemistry Committee and with their help, the Newsletter is able to offer information on trends in chemical education in most parts of the world. Topics in the current issue include a description of a Learning Aid Laboratory at Queen Elizabeth College, London, Physical Chemistry Teaching in Italian Universities, an account of "Lap Dissolve Projection at McGill University" - a new tool for learning and reports of discussions on Education in Biotechnology held in Berlin and on Chemical Education held in Wisconsin.

\section{Colour plates of t-RNA at $2.8 \AA$ recolution}

Two colour plates of t-RNA are being published with a full explanatory legend in the Journal of Molecular Biology, 1976, 166. 619.49, as part of a paper entitled "Crystallographic refinement of yeast phenylalanine transfer RNA at 2.8 R resolution" by $A$. Jack. G. E. Ladner and A. Klug. Since readers might like to have copies of the plates as a teaching aid, Biochemical Education has made arrangements to supply copies of the two plates at $\$ 1.00$ each including postage. They will not be generally available from the authors. The prints are expected to be ready by the end of January and orders (with payment) should be sent to Biochemical Education at 9 Hyde Terrace, Leeds LS2 9LS, England. 